Telecommunication Economics - Overview of the Field, Recommendations, and Perspectives

Stiller, B

DOI: https://doi.org/10.1007/s00450-009-0050-3

Posted at the Zurich Open Repository and Archive, University of Zurich ZORA URL: https://doi.org/10.5167/uzh-19501

Journal Article

Published Version

Originally published at:

Stiller, B (2009). Telecommunication Economics - Overview of the Field, Recommendations, and Perspectives. Computer Science - Research and Development, 23(1):35-43.

DOI: https://doi.org/10.1007/s00450-009-0050-3 


\title{
Telecommunication economics - overview of the field, recommendations, and perspectives
}

\author{
Burkhard Stiller
}

Published online: 12 February 2009

(C) Springer-Verlag 2009

\begin{abstract}
Telecommunication economics has evolved into an area of research, which includes technical aspects, social issues, economic and business factors, and regulation demands. The combination of those four dimensions is becoming critical for a suitable and viable understanding of communication needs in today's society. Therefore, this report on the Dagstuhl Perspectives Seminar "Telecommunication Economics"summarizes a number of key aspects of this field, it develops a set of selected and detailed recommendations, and outlines respective perspectives, which are being worked on partially already today and which need a much closer attention in the mid-term future.
\end{abstract}

Keywords Telecommunication and internet services .

Tariffing and pricing · Personalization - Incentives .

Value chain - Accounting - Contracts and legal domains .

Quality-of-Experience · Dynamic business · Competition

\section{Introduction and objectives}

The Dagstuhl Perspectives Seminar No. 08043 on "Telecommunication Economics" was organized to discuss and develop - at least - partially a strategic research outline among researchers active in that field, in order to enhance the knowledge on potential guidelines in the field of telecommunication economics and respective network management tasks for reaching integrated Internet and telecommunication networks in the nearer future. The view

\section{B. Stiller $(\square)$}

Department of Informatics, IFI, University of Zürich,

Binzmühlestrasse 14,

8050 Zürich, Switzerland

e-mail: stiller@ifi.uzh.ch on respective guidelines and recommendations to relevant players (end-users, enterprises, operators, regulators, policy makers, and content providers), focusing on the provision of new converged wireless services and content delivery networks to people and enterprises determined an aspect of relevance.

The main objective of this seminar was to allow business partnering to drive networking services and their sustainable provisioning for consumers and enterprises alike. This includes in more specific detail the following four objectives:

- The support of engineering leadership gained in mobile, broadband, digital TV, and wire-line communications, and selected media fields, by new sustainable business models in a fully deregulated and diversified demand framework.

- The study and identification of business opportunities throughout the value chain, especially for enterprises, content, and specialized services.

- The contribution to a strategy relative to socio-economic needs by increasing the motivation for deployment of cost effective and flexible solutions using networks and content.

- The provisioning of guidelines and recommendations for utilizing different types of technologies and quantify necessary actions. These results will potentially supply regulators and standardization bodies with analysis and guidelines for creating conditions for fast growing competitive mobile, broadband, and content markets while speeding up business.

Thus, in a nutshell, the "Telecommunications Economics" Seminar shall help researchers to guide the development of (a) applicable and usable prototypes and (b) relevant services from the socio-economic, technical, and business perspectives. 


\section{Overview of the field}

The telecommunications sector and the Internet section of Internet Service Providers (ISP) have become a dynamic key area for the economic development of industrialized nations in the world. It is in constant evolution. Because of intense competition, telecommunications companies and ISPs are forced to diversify their offers and thus to propose an increasing number of services. However, on one hand, economic analysis often ignores important technical aspects of telecommunications and is not aware of new developments. On the other hand, engineering models often ignore economic factors. Thus, the design and deployment of future networks that incorporate new services are subject to uncertainties such as equipment and capacity prices (due to technological innovation) or demand and supply for services (due to competition). Seeing leading researchers bringing together with various backgrounds, all working on innovative aspects of technical, techno-economic, socio-economic, and regulatory issues, lead to the following four main areas that have been - partially - tackled in an integrated and combined manner:

- Architectural side,

- Social side,

- Economic and business side, and

- Regulatory side.

\subsection{Architecture}

With respect to the architectural side, IP (Internet Protocol)enabled Next Generation Networks (NGNs) have gradually become reality creating numerous challenges for all ICT (Information and Communication Technology) sector stakeholders. Traditional network operators, attracted by many advantages offered through NGN solutions, have decided to migrate to unified networks that enable future electronic communication based on IP. Many stakeholders, already realizing the economic potential of the NGNs, have begun implementing necessary multi-million dollar investment programs, even when the potential business models and future regulatory framework are still under discussion. The variety of architectures, e.g., all-IP networks, and technology, e.g., fixed networks, wireless access networks, and mobile user support, need to be determined and analyzed.

Finally, determining the general link into the economic and business side, at this stage of the development it is still difficult to predict business models - based on the economics of information and communication infrastructures that will certainly set new standards and revolutionize the communication sector in the future. The efficiency and effectiveness of such models can only be verified through market mechanisms that will be substantially changed in the new converged environment.

\subsection{Social issues}

On the social side, end users mandate the capability to establish and develop their people's interests and content networks, driven by personal fulfillment and without affordability constraints. They may want to set own price and service configurations and to join community networks, all leading to complex agreement structures with all involved suppliers.

With respect to an emerging application of content delivery most content providers, typically allied sometimes with network providers, prosper on what is essentially a pure "push" model fuelled eventually by advertisements or subscriptions. This mass distribution concept is also a very powerful tool to uniform the culture and it gives excessive power to these centralized information distribution players. Thus, the social side becomes highly relevant. Research is needed into the socio-economic implications of the reverse "pull" model, also fuelled eventually by advertisements, subscriptions, and the supply of personalized content. And another emerging content delivery model is the "personalized push model", which is enabled by the mobile communication technology. In this model, selected content is delivered to a certain segment of users.

In another important application domain of social studies, e-health or health telematics, the social aspects of society's development need to be perfectly accounted for and IT as well as research networks deployment has to be extremely cost-effective in a mid-term scenario. Namely by using ICT support for tele-medicine, distance learning for health care service providers, and access to medical data, social questions arise.

Amongst others, the following issues require a socioeconomic elaboration: a disaster management network with exact planning and monitoring, a quality control network and monitoring tools, and electronic patient records and e-Health cards for citizens. Additionally, the "co-opetition" foreseen, mainly between the various players in the sample application area of e-health, will be able to show the interactions of different stakeholders.

\subsection{Economics and business}

Concerning the economic and business side, for most businesses and public sector entities communications services are purchased for several years based on traditional competitive bidding. This is far from companies' real desire to use networks in their business strategies, with fast connect/fast disconnect schemes to temporary partners, which has been researched in the field of smart business networks. 
Companies want also to assemble dynamically network hardware and software components to serve and control multi-party business processes. Much research is still needed into multi-party Service Level Agreements (SLA), authentication, signaling, and privacy with an integral focus also on those related legal aspects and liabilities. Business processes, such as Customer Relationship Management (CRM), Enterprise Resource Planning (ERP), payments, or service fulfillment, require companies themselves to set up not just traffic models, but information models also. Additionally trust and customer identification are highly essential. Finally, there are also concerns about today's legal aspects regarding the sharing and distribution of user generated content.

\subsection{Regulation}

On the regulatory side, technology neutrality is an established principle, but business roles are still a major issue. The separation or overlap of deposit banks and network operators is no longer justified. Just like sooner or later utilities will want to play a role in the last mile access. However, research is needed, including on the legal side, to see how public interest is best served in such initiatives. Furthermore, prospects for telecommunication regulation based on dynamic economic theory become essential, since the business models develop further and rapidly changing alternatives and choices need to be addressed and adapted in a legal framework. Thus, a comparative analysis of regulatory models, originating in EU or US regulatory guidelines and country-specific guidelines, is important to provide for a clear and concise basis, which can be used by providers of telecommunication services, data services, and content services as well as by customers.

\subsection{Discussion of an integration}

Telecommunication Economics outline a key aspect of a commercialized networking world, covering telecommunication networks as well as the Internet, which address a.o. the pricing problem for Internet services and various management as well as resource allocation problems under economic perspectives.

The combination of (a) technical mechanisms in terms of architectural considerations, (b) social-economic aspects in terms of individualized services and tariffs., and (c) economic models determines the best possible methodological approach for optimizing the commercial operation of services in the heterogeneous world of wired and wireless networking technology environments. Furthermore, (d) the legal and regulatory points of views of regulations and contracts lead in this perspectives seminar to the emphasis on observable trends of a continuous consolidation in players and an oligopoly situation on the global scale, which most likely will remain with some limited global players on the telco side.

Application-wise the wide range will continue to be increased with new services and approaches, where the consumer will always try to maximize his surplus, where the social planner tries to maximize social welfare, and where operators will try to maximize their profit. Thus, the triangular set of forces will act in the electronic services market as the world has seen actors in the physical goods markets. Only time-scales observed will be much shorter and highly interactive, since the offer of choices will be extremely broad, changes will happen with a high frequency, and technologies evolvement will foster new business opportunities and challenges for the almost ever chasing regulatory and legal issues in telecommunications.

\section{Dedicated scientific contributions}

The seminar was organized in three sessions, addressing the following topics:

- Value Chains and Personalization,

- Dynamics in Accounting, Contracts, and Virtuali-zation Effects,

- Motivation and Incentives in Mesh Networks, Pricing Schemes, and Use Cases.

The set of presentations given and their main ideas as well as the key content is summarized below, while the outcome of discussions following these dedicated approaches are summarized in Recommendations and Perspectives afterwards, resulting in guideline proposals and recommendations to be considered.

\subsection{Value chains and personalization}

The first set of topics covered the problems of valuation of mobile service usage, of an individualization of tariffs for mobile services, and the discussion of possible players' changes in the value chain of telecommunications. Thus, in this respect many social aspects are combined with architectural questions as well as respective economics.

\subsubsection{Value of mobile service usage}

It has become possible recently to measure the usage of services by mobile subscribers very accurately. This new level of accuracy is based on a data measurement directly collected from mobile handsets. This process allows to capture all user transactions, such as timestamp, application, uplink/downlink data, or location. This data is highly relevant for estimations, which are performed, of the value of a us- 
age per transactions. Notably there is no way to get direct price tags from anywhere else. The best data available today is time-based. Therefore, the essential question is: How to turn time into value?

\subsubsection{Individual mobile communication services and tariffs}

Individual services and tariffs existed in the beginning of telecommunications history already 150 years ago but have faded away over time. Service provisioning evolved into the current supplier-centric situation, which has many limitations and disadvantages. Therefore, the user-centric service provisioning and its application to the current mobile communication service setting is done, which, however, differs significantly in scale and scope from the historical practices. A design methodology and tool for the determination of individualized mobile services and tariffs is provided and its benefits to both the user and the supplier are evaluated.

The design has three aspects. The first involves the construction of a conceptual framework consisting of behavioral models of the user and the supplier (firm) and a game theoretical negotiation mechanism to determine individual services and tariffs. The second aspect covers the operationalization of the conceptual framework in a computational design with methods, computational models, negotiation algorithms, risk metrics, and a prototype implementation. Thirdly, the extension of individual services and tariffs concept is done to achieve a community setting via a community business model proposed.

Two evaluations are performed. For the firm-based design, a user survey is conducted and computational cases, which address value-added mobile services and generic mobile service bundles, are developed. Numerical analyses show that users always achieve gains in utility. Benefits to the supplier include adjustable risk-profit equilibrium points, increased network traffic, and reduced churn. For the community-based design, two case studies on communities are conducted. These results demonstrate that the proposed business model of community-based individual services provisioning and tariffing can meet demands of their members precisely and address both affordability and sustainability issues.

Finally, a specific engineering and integration of individualized service and tariff design tools into existing infrastructure of communication services suppliers is proposed.

\subsubsection{Going down the value chain?}

Ten years ago telecommunication companies (telcos) claimed that it would become impossible to earn money with transferring data and that it would be necessary to move up in the value chain and sell content, like music and video.
After ten years it may be concluded that telcos failed to move up in the value chain, despite enormous amounts of money that were invested into their infrastructures and content business.

The question is now whether this situation, in which control over network and contents is in different hands, is really stable. Will there never be a single organization controlling network and contents?

The answer to this question is unknown yet, but examples like Google indicate that the opposite may happen of what telcos aimed at: content providers move down in the value chain and might take over control over (parts of) the network, which is technology-wise doable. However, will it be economically viable?

\subsection{Dynamics in accounting, contracts, and virtualization effects}

The second set of topics covered an accounting approach for a fully dynamic service environment. Additionally, the need for legally binding contracts for service delivery has been stated, which has to be supported in a dynamic situation across legal domains. Finally, the dynamic demand for bandwidth in a virtualized network is discussed. Thus, many aspects of economics and a technical architecture are highly inter-related as well as legal questions are put forward.

\subsubsection{Accounting, charging, and billing in dynamic business environments}

The increasing dynamicity of business environments (consumers being producers, short-lived relationships, unknown parties, or partner settlements) entails a big challenge for the Accounting, Charging, and Billing (ACB) process between interacting business parties. The work presented takes the broader view of compensation, first coined in the Ambient Networks Project (FP6-2006-IST-027662-AN P2), which covers negotiation and implementation of business agreements as well as the exchange of values (monetary and non-monetary) between parties.

The key requirement in a dynamic business environment is the support of an automated and dynamic negotiation of compensation agreements with parties known or unknown to each other. Moreover, according to the negotiated agreements (e.g., including price, payment method, or compensated and compensation party) the compensation functionality has to be implemented in an automatic way. Finally, a compensation system must be able to handle heterogeneity in case of integrating different or legacy compensationrelated components during its implementation phase.

In this a functional compensation architecture is described, which comprises out of an Operation Subsystem, a Control Subsystem, and an Interaction Subsystem. The 
architecture was validated by a prototype, which is applicable to upcoming application domains, such as congestion pricing, decentralized energy production, and Software-asa-Service (SaaS) supply chains.

\subsubsection{Legal compliance in commercial service provisioning across administrative domains}

Internet design principles originally do not focus on commercial service provisioning. Hence, in a commercialized world of service offerings support mechanisms need to be implemented in order to ensure that value-added services can be offered in a competitive context. Commercial product offerings base on contractual agreements concluded between service providers and service customers. Contracts need to reflect business-driven requirements originating from involved contract parties, while they are invariably required to respect those regulations imposed by commerce law.

Legal compliance, thus, determines the available range of applicable contractual terms - irrespective of whether such a contract governs commercial value-added services in the Internet or not. Typically, legal determinations are valid in a limited geographical area only. The Internet, however, lacks an exact distinct notion of such a location. Consequently, technical means to overcome this fundamental design gap are investigated, in order to ensure that legally compliant contracts can be determined, set-up, and settled.

\subsubsection{Network virtualization as a key driver for bandwidth markets}

Recent technical advances in telecommunications, especially in the area of optical fibre technology, have led to a tremendous increase of bandwidth, based on which the support of Quality-of-Service and diverse Internet-based application services such as VoIP and IPTV became possible. However, suitable bandwidth trading mechanisms for such services, which require in many cases short-termed bandwidth assignments, e.g., for large sporting events or cultural open air activities, have not yet evolved. In particular, there is currently no technically and economically feasible solution in place, enabling service providers to buy necessary bandwidth for such services "on demand" and resell it to other providers if not used.

This work presents how PeerMart, a fully decentralized auction-based marketplace based on peer-to-peer networking principles, can be applied to trade bandwidth services in an efficient and scalable manner. A specific scenario is developed, targeted at trading bandwidth over optical links in a fully virtualized networking environment, from which detailed requirements are derived. Particular technical and economic aspects of the scenario are investigated and spe- cific service parameters defined. Finally, PeerMart's basic design supporting generic services is refined to meet those specific requirements of the considered bandwidth trading infrastructure.

\subsection{Motivation and incentives in mesh networks, pricing schemes, and use cases}

The third set of topics covered a motivation for incentives provided by technology and pricing schemes. While cross-layer incentives are discussed for mesh networks, a Quality-of-Experience path out of the flat rate pricing trap is outlined, which is complemented by a use case study of mobile telephony prices in Poland, determining regulatory goals. Thus, economics are again closely inter-related with regulatory requirements, being backed by a number of certain architectural assumptions of the networking infrastructure.

\subsubsection{Cross-layer incentive mechanisms: the case of self-organized neighborhood wireless mesh networks}

The potential of self-organized Wireless Mesh Networks in the neighborhood is shown in this work as well as the impact of the existence of such networks in telecommunication economics, as done within the FP6 WIP project (An All-Wireless Mobile Network Architecture). The most challenging issues toward this context include (a) the desire of users to participate in the first place, (b) the ability to form trustworthy links at the network layer, and (c) to explain suitable incentives for resource sharing. It was argued that a promising approach in this direction is the design of a community-aware network architecture, technology-aware social incentives, and cross-layer incentive mechanisms.

\subsubsection{Out of the flat rate trap? On the role of quality-of-experience for charging communication services}

Over the last 10 to 15 years, Quality-of-Service (QoS) has turned out to be an eternal source of inspiration for all types of Internet pricing. At the same time, it has been observed that the scope of QoS research has been more and more limited by a purely technical scope, in contrast to the original intention. Therefore, more recently the interest in "Quality-of-Experience" (QoE/QoX) has brought back the end user-control perspective.

This work undertaken provides a brief survey of current understanding and modeling of QoX and presents initial approaches how to link it to Telecommunication Economics. In this sense, the aim is to get a first answer to whether charging should become part of the future QoX research agenda or not. 


\subsubsection{Mobile telephony in Poland - competition and prices}

The purpose of this study is to find an answer to the question of whether the Polish mobile market is competitive enough to provide maximum benefit of users in terms of the variety, prices. and quality of the service provided, which is among the most important aims of regulations of the Polish Telecommunications Law. It is also worth to consider whether the present provisions of the law suffice to achieve another aim deduced, i.e. supporting of a fair and efficient competition in the matters of provision of telecommunications services.

\section{Recommendations and perspectives}

Based on those three sessions described above and the four main areas of work introduced in Sect. 2, the discussion was guided by three questions, the perspectives seminar was supposed to take into close account:

- In which way and with which engineering areas of work will a possible future and up-to-date network service offer become successful?

- What are the major fields to be considered for allowing a consolidation of business opportunities for operators, customers, and end-users at the same time?

- In which form and with which applications does a consolidation of socio-economic needs happens, enabling a fair service offer, use, and payment?

\subsection{The engineering point of view}

In consolidation of those engineering issues, which address the technology in use for converging services in telecommunications and the Internet, a number of dimensions are considered to be of importance:

- The strategic and operational value of IPv6 (Internet Protocol Version 6): Relevant economics need to be differentiated by the region, however, a driver for Europe, Asia, and the North American market are foreseen.

- Competitive alternative wireless infrastructures: A wide market share with different quality levels, possibly agentbased systems will go beyond the state-of-the-art ad-hoc networks known today. In particular, vehicular networks as well as e-health applications for a mobile society will strengthen mobile technology developments.

- Mass customization engineering: The need to address a single user and his or her very specialized requirements, profiles, and service demands will support the convergence of telecommunications and Internet-based communications, while the tariffing of end-users and smaller groups of combined users demands for suitable and scalable accounting, charging, and tariffing mechanisms.

- Regulation: The need for a service compliance to legally determined regulative bylaws and acts leads to the emerging demand of automated and scalable mechanisms to audit, e.g., a Service Level Agreement (SLA) negotiated has to be compared to the current level of service delivered.

- Telematics: The role of communicated information across physical locations and countrysides is crucial for an information technology-based society. E.g., in-car technology and its inter-operation with road safety systems, the supervision of distributed sensors for human health and the environment, and Green Information and Communication Technology (ICT) in a much more general form do open new opportunities.

- Data analysis: The knowledge about data and its origins as well as suitable data mining algorithms opens the path for valuable and dangerous applications. Those include (a) the tracking of a person's curriculum vitae and parts of it in the Internet, where tasks, issues undertaken, as well as locations, where the person has been to can be are included and (b) usage pattern analysis of networks and services for optimizing network operations within a given network management system. In all of these cases, the privacy aspect of data being analyzes determines a high level of functionality to be achieved.

- Traffic management: The need for an efficient and effective traffic management beyond network boundaries, including overlay traffic and peer-to-peer traffic, becomes obvious as soon as their appearance in networks reach a non-marginal percentage. The goal to optimize this network's bandwidth in a win-win-type of situation for both the operator and the end-user at the same time determines a challenge.

- Next Generation Internet Architecture: While the traditional FTP/Telnet-based Internet service model has been operational for about three decades, the successors of these services, even leaving the Web and Web 2.0 behind, include facebook, peer-to-peer systems, Qualityof-Experience (QoE/QoX) support, and Bandwidth-onDemand mechanisms, are awaiting a growing demand from end-users. Thus, relevant and necessary changes to the Internet's architecture are a must in a short-term.

- Green Telecommunications: There are emerging needs to investigate on how to use telecommunica-tions technologies to achieve a greener environment, to reduce the $\mathrm{CO}_{2}$ emission as well as other environ-mental pollution. This will be a research issue in combination of socialeconomic, techno-economic, and technical areas, which does effect design, development, and production processes in the same way as the installation, operation, and maintenance $f$ these ICT products in society. 


\subsection{The business point of view}

Secondly, the consolidation of business opportunity issues is essential to determine a multitude of approaches, which cover operators, customers, and end-users in a fully integrated requirements' analysis as well as a prospective set of service opportunities.

- Value chain: The positioning of telecommunication and Internet providers, third party providers, and users in the value chain has to be reconsidered. While the traditional telco undergoes a strong change to be able to survive economically in a country, region, or the world, third party service providers (a.o. on virtualized service offerings) seem to take advantage of these opening opportunities. A definition of new segmentations of these roles and their responsibilities seems to be unavoidable.

- Open Source Principle: As known from the software business, open source software is driven by a community of people interested in a certain package, functionality, or ICT system. The question remains in which way a possible monetization of voluntary work, a customerdriven customization of this software, and combination of open source with closed source software will benefit a networked collaboration. While communities in terms of Wireless Mesh Networks support the open use of networking capacity as such, an unleash of mobile customers and the innovation power of the wireless domain will develop further applications, such as e-health and support of the elderly living.

Furthermore, there is the need to analyze the benefit and risk of open source to mobile networks and its technologies. Currently, the mobile network technology in the wide area is "closed", and operators have tight control of it. While the open source approach may bring security problems, such as viruses or hacking, to the network, the openness in terms of value-added third party services may increase due to new business opportunities for small and medium-sized enterprises.

The same principles may apply to open source hardware, where handheld computers and PDAs (Personal Digital Assistant) as well as routers for inter-networking may see valuable contributions to a maintenance and development opportunity. Opening the handset has proven to offer a highly accepted platform for a variety of newly developed applications. Of course, the i-mode examples shows that in a certain type of market closed systems work well, too.

- Services: The pure offer of services will not guarantee any success. This is fully acknowledged for the transport of bits (bit-pipe), which does not determine any viable business model today any more. However, the value addition to services, either offered from the telco or In- ternet service providers or even from third parties (such as overlay application providers or content providers), gives raise to a number of potential opportunities. Those include the support and maintenance of services, their adoption to new domains and applications, and their continued development to embed new underlying technologies and advances in networking.

- Identity Provider: The prevention of electronic misuse of electronic identities is a key issue to be tackled. While the management of identities within a single domain is solved, the multi-domain-based identity provisioning and maintenance remains a problem due to the lack of standards, registration mechanisms, and efficiency aspects. The opportunities will raise as soon as multi-domain solutions are available, which will foster the use of electronic service provisioning.

- Trusted Computing Platform: While this type of underlying technology was considered a must in terms of mechanisms of high use, on one hand, the efficiency, security holes, and production process in a proprietary as well as expensive manner was, on the other hand, considered to be a major drawback.

- Payment Provider: Although the set of electronic payment schemes in place have seen a certain degree of revival, the non-standard set of approaches seem to thwart the overall success. However, the need for an open, efficient (in terms of transaction costs) and secure micropayment scheme remains and will offer opportunities for a variety of services.

The role of payment providers based on federations and applying SAML (Security Assertion Mark-up Language) extensions will see an increased usage in multi-domain, roaming situations, since already existing and operational functionality will require a minimal extension only to become operational in a multi-domain scenario.

- Bandwidth-on-Demand (BoD): The set of existing applications requiring VBR (Variable Bit Rate) services dominates CBR (Constant Bit Rate) services. Thus, especially short-term fluctuations in bandwidth demand, either on the physical layer (including optical and electric network technologies) or on the transport/IP layer, are important to be managed in a fully automated manner. BoD control mechanisms will enable VBR applications to come and go in a much higher frequency (viewed from the providers' side) and will obtain a much better quality experienced by the customer, which determines a valuable opportunity.

- Regulation: While regulation omits - on purpose - market forces to play, it prevents at the same time monopoly and oligopoly situations from shearing a competitive pricing. However, regulation may limit the development of new services and offers at the same time, which have not been possible at the time of their set-up. Thus, the 
opportunities cover the definition of regulatory policies, which encourage players to join the market, but which prevent monopolies to come up due to dominating market positions.

\subsection{The socio-economic point of view}

Finally, the consolidation of socio-economic needs of telecommunication and Internet service users determine a key aspect for a fair and balanced society, taking into account different groups of users and different types of providers. Thus, a number of dimensions are considered to be of major importance.

- User-generated content and services: The trend to see traditionally consuming users being able to provide content changes traffic and usage patterns of today's networks. The society, however, needs to respond to the question, is there a surplus? If so, the need for suitable decentralized content ratings, content quality checks, and reliable content management schemes arises.

- Quality/value ratio: Besides those technical and economic mechanisms being developed to reach a true valuation and delivery of Quality-of-Experience (QoE/QoX) a user is seeing, the set of metrics required to rate, a.o., the time value, to access the perception of services, or to determine the content's usability need to leave subjective grounds. Thus, objective and standardized mechanisms and schemes (e.g., going clearly beyond telecommunication-based approaches for phone calls) need to be developed for a wide range of network-based services.

- Application and user segment: The crucial field of socioeconomic-dependent applications include health services and the support of elderly living. Thus, the so-called "Silver Economy" - assuming that for a larger portion of elderly people tomorrow the financial situation in the mid-term future will partially enable necessary and entertaining telecommunication services in a broader scale -, neighborhood communities, and dating software as well as social software show an inherent societal aspect of responsibility and life-supporting (or life-threatening) potential. Its effects on community, governmental, and private sector funding schemes need to be studied.

- Fairness of prices and services: The human-observed, sometimes relative however, perception of privileges, service qualities, and discriminating prices will effect the businesses/prices/customer relation management. In particular, today the roaming fees/prices/rates area of concern (for regulators, governments, operators, and customers) serves as a good example for necessary models and solutions to overcome mutual allegations. The "open access to information" principle as well as different anticensorship trends complement the fairness problem.
- Morality: The social responsibility of service providers to offer prices for telecommunication services, which show a realistic and traceable background, has to be emphasized. While users' behavior may be determined by honest or malicious factors, incentives for undermining legal boundaries of service usage, mainly in terms of reuse and reselling, have to be minimized by fair prices.

- Legal bodies: The situation of multi-domain service offers and consumptions stretches across multiple different areas, typically geographical areas of legal domains, such as the EU, Switzerland, or the US. Therefore, the need for an Internet Law deems to be useful under a certain perspective. However, legal situations of countries and unions considered as well-established legal domains have to be taken into account as well.

- Reduction of complexity: The use and application of ICT faces the crucial Human Computer Interface (HCI) areas, in which essential relaxations are required to achieve: a.o., transparencies of service characteristics and prices, easy-to-use functionality (e.g., the red button example), and an embedded service usage situation, which does avoid a traditional user technology reluctance.

\subsection{Perspectives}

In the mid-term range Telecommunication Economics will be effected by law and policy guidelines, which will vary depending on the region of the world, where services are offered, consumed, or maintained. This regionalized view point may enable a simpler understanding of those problem areas addressed, though, it defines an important obstacle for a world-wide and fully open market, unregulated operating behavior of those players considered. Under the same set of rules, regional approaches, such as within the European Community or the North American countries, will become successful. However, just to name a few, (a) the need for multi-domain work, crossing boundaries of legal domains, (b) the need for interdisciplinary research work, especially on effects of the right incentives for service delivery, proof, and provisioning, and (c) the need for service valuations and personalization do determine important problems has been determined - and for those a look into suitable, efficient, effective, and multi-national solutions is highly demanded.

A number of those areas tackled in the Perspectives Seminar on "Telecommunication Economics" are being worked on already in a variety of national and European projects (cf. Acknowledgements). Therefore, this Dagstuhl Perspectives Seminar did foster a high degree of interaction and collaboration among participants from major fields of research, addressing Telecommunication Economics. However, the seminar concludes as well that those views expressed above 
neither may be complete nor may they be fully in line with many companies' professional goals as they are stated today. Thus recommendations given are selective, based on good grounds of knowledge.

Acknowledgement This report was made possible due to the partial support of the COST Action Econ@Tel: A Telecommunications Economics COST Network (IS0605), the FP7 STREP SmoothIT: Simple Economic Management Approaches of Overlay Traffic in Heterogeneous Internet Topologies (FP7-2008-ICT-216259-STREP), and the FP6 NoE EMANICS: European Network of Excellence for the Management of Internet Technologies and Complex Services (FP6-2004IST-026854-NoE). In particular the following people - in alphabetical order - provided input to this report: Panayotis Antoniadis, Hong Chen, Heikki Hammainen, David Hausheer, George Huitema, Jerzy Kubasik, Aiko Pras, Peter Reichl, Burkhard Stiller, and Martin Waldburger.

\section{References}

1. Econ@Tel Home Page (2008) COST Action IS0605: http://www.cost605.org, December 2008

2. EMANICS Home Page (2008) http://www.emanics.org, December 2008

3. Hausheer D, Nikander P, Fogliati V, Wünstel K, Angeles Callejo M, Ristol Jorba S, Spirou S, Ladid L, Kleinwächter W, Stiller B, Behrmann M, Boniface M, Courcoubetis C, Li M-S (2008) Future Internet Socio-Economics - Challenges and Perspectives. White Paper, Future Internet Assembly, Madrid

4. Heikkinen MVJ, Matuszewski M, Hammainen H (2008) Scenario planning for emerging mobile services decision making: mobile peer-to-peer session initiation protocol case study. Int J Inform Decision Sci 1(1):26-43

5. Kubasik J (2005) The Polish Cellular Telephony Market: Is It Competitive Enough? 16th Regional European ITS Conference, Porto, Portugal, September 4-6

6. Pras A, Schönwälder J, Burgess M, Festor O, Martinez Perez G, Stadler R, Stiller B (2007) Key research challenges in network management. IEEE Commun Mag 45(10):104-110
7. Reichl P (2007) From Charging for QoS to Charging for QoE: Internet Economics in the Era of Next Generation Multimedia Networks. 1st International Conference on Autonomous Infrastructure, Management and Security (AIMS 2007), Oslo, Norway, June 21-22, 2007, Lecture Notes in Computer Science Vol. 4543

8. Rietkerk O, Huitema G, Markendahl J (2006) Business Roles Enabled by Ambient Networking to Provide Access for Anyone to Any Network and Service. Helsinki Mobility Roundtable, Finland, June 1-2, 2006

9. SmoohtIT Home Page (2008) http://www.smoothit.org, December 2008

10. Stiller B (Ed) (2008) Joint EC-GIN, EMANICS, and SmoothIT Workshop on "Economic Traffic Management". University of Zürich, IFI Technical Report, No. ifi-2008.10, August 2008

11. Waldburger M, Stiller B (2007) Legal Compliance in Commercial Service Provisioning Across Administrative Domains. 13th Open European Summer School and IFIP TC6.6 Workshop on Dependable and Adaptable Networks and Services (EUNICE 2007), Enschede, The Netherlands, July 2007

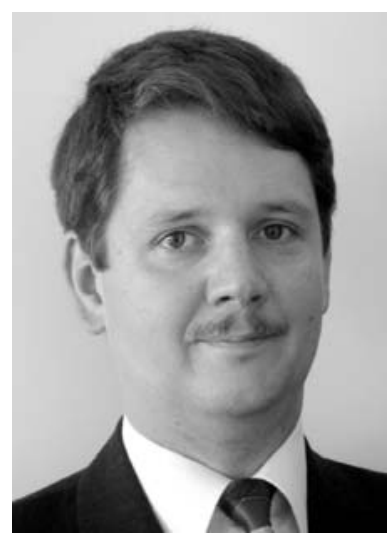

Burkhard Stiller Prof. Dr. Burkhard Stiller chairs the Communication Systems Group CSG, Department of Informatics IFI at the University of Zürich UZH, Switzerland since 2004. He holds a Computer Science Diplom (October 1990) and a Ph.D. degree (February 1994) of the University of Karlsruhe, Germany. During his previous research locations at the Computer Laboratory, University of Cambridge, U.K., the Computer Engineering and Networks Laboratory, ETH Zürich, Switzerland, and the University of Federal Armed Forces, Munich, Germany his main research interests cover, including current CSG topics, charging and accounting of Internet services, economic management mechanisms, systems with a fully decentralized control (P2P), telecommunication economics, and biometric management systems. 